\title{
Analysis on the Influence Degree of Processing Quality Parameters on Bearing Vibration (Part II : Experiment)
}

\author{
Xintao Xia ${ }^{1, a}$, Xiangfeng Chen ${ }^{1, b^{*}}$, Xiaowei Yang ${ }^{2, c}$ and Bin Liu ${ }^{1, d}$ \\ ${ }^{1}$ Mechatronical Engineering College, Henan University of Science and Technology, Luoyang \\ 471003, China \\ ${ }^{2}$ Luoyang Bearing Science \& Technology Co., Ltd., Luoyang 471039, China \\ axiaxt1957@163.com, bblack_xfc@163.com, yangxwzys@163.com, dIbin1992@foxmail.com
}

Keywords: Bearing vibration; Fuzzy set theory; Processing quality parameter; Influence degree

\begin{abstract}
In order to obtain the main influence factors of bearing vibration, based on correlation analysis of fuzzy set theory, channel radius of curvature, channel shape error and channel surface roughness are selected as processing quality parameters to research the influence degree of different processing quality parameters on bearing vibration. The inner ring channel shape error and the outer ring channel surface roughness as the main factors affecting the bearing vibration are finally drawn.
\end{abstract}

\section{Introduction}

Bearing vibration is a complex mechanical phenomenon [1]. The reason for the vibration is also complicated. Ismail, MAA studied the vibration response characteristics of off-ball bearings [2]. Tse, Peter W studied the state space of the nonlinear vibration response collected by the dynamic rotor bearing system [3]. Prudhom, A analyzes the time-frequency vibrations of motor damage caused by bearing current [4]. Guo, Jun-Feng studied the data compression method of collecting vibration signals of rolling bearings [5]. Although scholars at home and abroad are committed to research in this field and some achievements have been made, the total bearing vibration theory has not yet been established [6]. Considering the difficulty of statistical analysis, this paper adopts the method of correlation analysis in fuzzy set theory.

In this paper, the Average membership degree, Euclidean distance and Closeness degree in fuzzy set theory are combined with qualitative fusion and the main factors affecting the bearing vibration can be effectively filtered out, which provides a new theoretical basis for controlling and improving the quality performance of the bearing. This has a very important practical value.

\section{Experimental Study and Data Analysis}

The symbols used in this paper and their meanings are listed in Tab. 1. The data obtained from the experiment is shown in the following figures.

Table 1 Symbols and manings

\begin{tabular}{|c|c|c|c|c|}
\hline Serial Number & Symbol & Meanings & $\begin{array}{c}\text { Parametric } \\
\text { Performance }\end{array}$ & Unit \\
\hline 1 & $X_{0}$ & Vibration acceleration effective value & Performance & {$[\mathrm{dB}]$} \\
\hline 2 & $X_{1}$ & Inner ring channel radius & Structure size & {$[\mathrm{mm}]$} \\
\hline 3 & $X_{2}$ & Outer ring channel radius & Structure size & {$[\mathrm{mm}]$} \\
\hline 4 & $X_{3}$ & Inner ring channel shape error & Macroscopic shape & {$[\mu \mathrm{m}]$} \\
\hline 5 & $X_{4}$ & Outer ring channel shape error & Macroscopic shape & {$[\mu \mathrm{m}]$} \\
\hline 6 & $X_{5}$ & Inner ring channel surface roughness & Microstructure & {$[\mu \mathrm{m}]$} \\
\hline 7 & $X_{6}$ & Outer ring channel surface roughness & Microstructure & {$[\mu \mathrm{m}]$} \\
\hline
\end{tabular}

The data of baring A is shown as follow. 


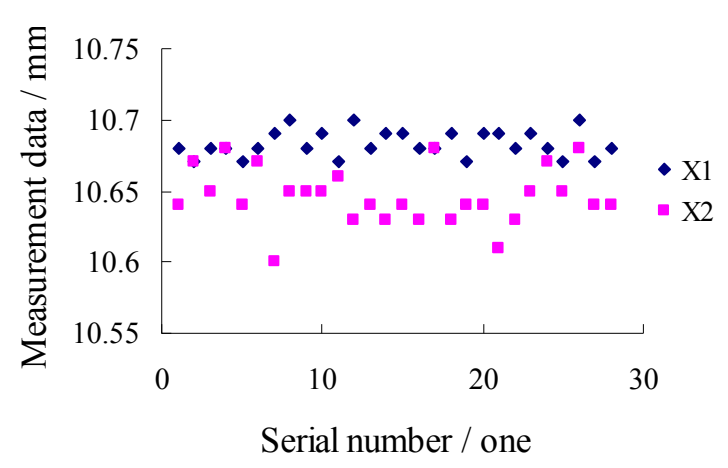

Figure 1. Inner and outer ring channel radius

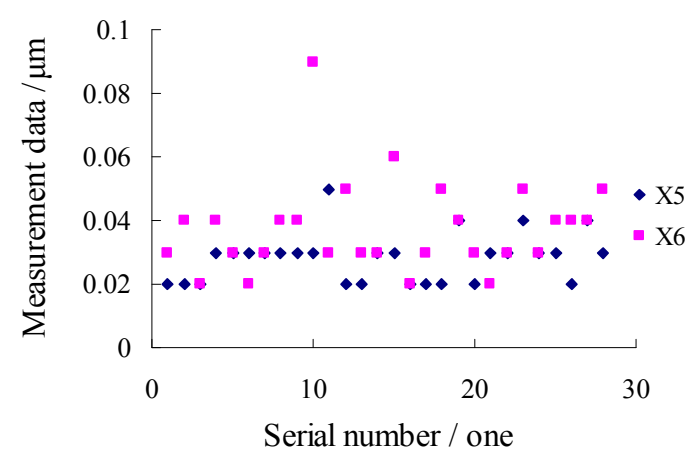

Figure 3. Inner and outer ring channel surface roughness

The data of baring B is shown as follow.

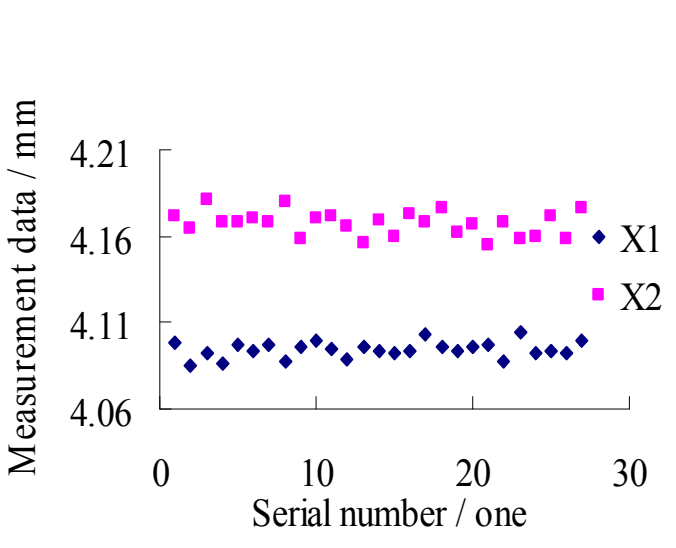

Figure 5. Inner and outer ring channel radius channel radius

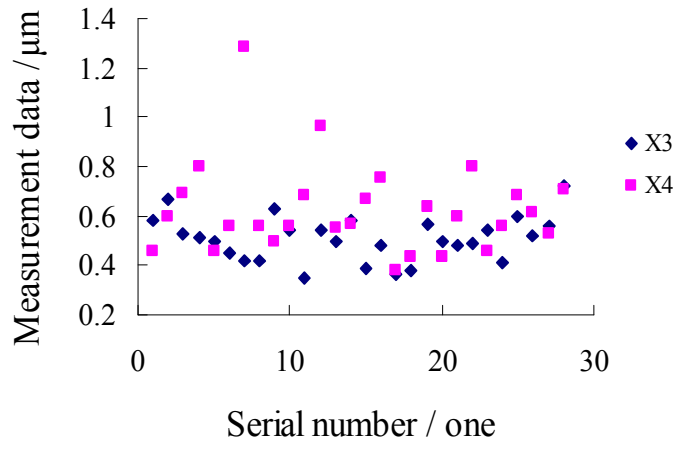

Figure 2. Inner and outer ring channel shape error

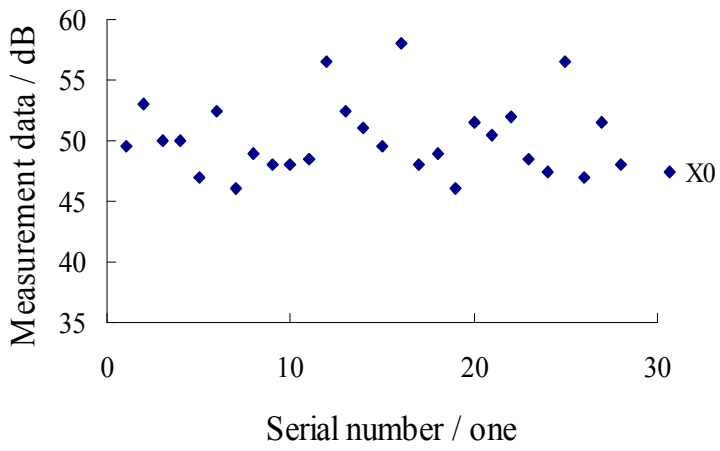

Figure 4. Effective value of vibration acceleration

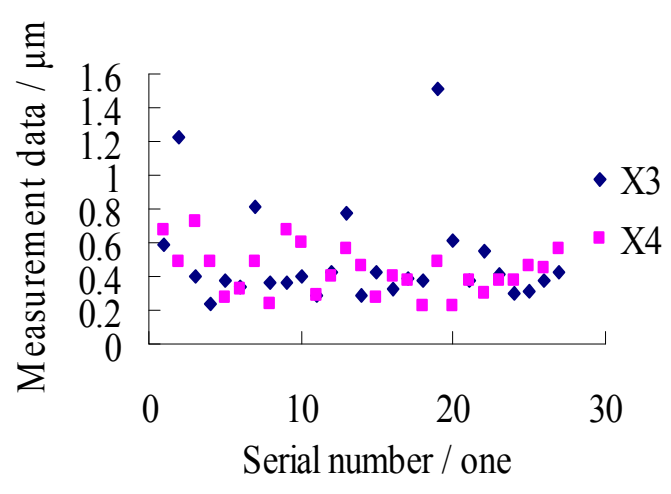

Figure 6. Inner and outer ring channel shape error 


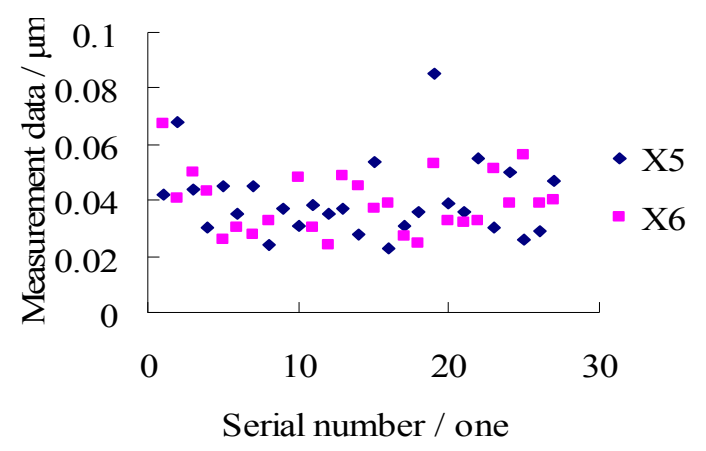

Figure 7. Inner and outer ring channel surface roughness

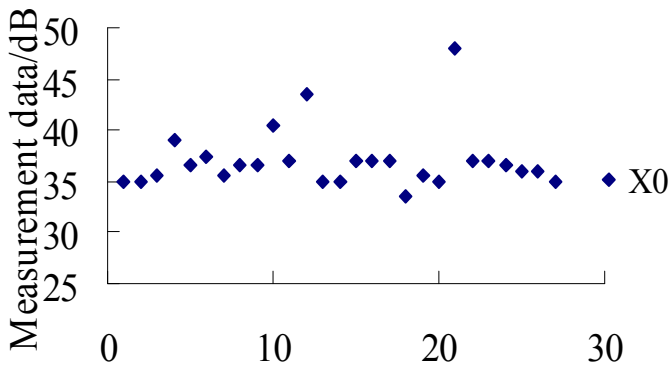

Serial number / one

Figure 8. Effective value of vibration acceleration

According to the relative size of the Average membership degree $\mu_{k}^{*}[7]$, the influence degree of the Processing quality parameters $X_{j}$ on the bearing vibration $X_{0}$ is evaluated. The results are shown in Table. 2.

Table 2 Average membership degree $\mu^{*} \mathrm{k}$

\begin{tabular}{|c|c|c|c|c|c|c|}
\hline Bearing Number & $\mu_{1}$ & $\mu_{2}$ & $\mu_{3}$ & $\mu_{4}$ & $\mu_{5}$ & $\mu_{6}$ \\
\hline $\mathrm{A}$ & 0.4640 & 0.4301 & 0.5557 & 0.6359 & 0.4560 & 0.4891 \\
\hline $\mathrm{B}$ & 0.3758 & 0.3827 & 0.5681 & 0.4826 & 0.5378 & 0.5376 \\
\hline
\end{tabular}

According to the above table, for bearing $\mathrm{A}$ and $\mathrm{B}$, these are

$$
\begin{aligned}
& \mu_{4}>\mu_{3}>\mu_{6}>\mu_{1}>\mu_{5}>\mu_{2} \\
& \mu_{3}>\mu_{5}>\mu_{6}>\mu_{4}>\mu_{2}>\mu_{1}
\end{aligned}
$$

According to the relative size of Euclidean distance $d_{p}$ and Base-based Closeness degree $N[8-9]$, the influence degree of the Processing quality parameters $X_{j}$ on the bearing vibration $X_{0}$ is evaluated. The results are shown in Table. 3 and Table. 4.

Table 3 Euclidean distance $d_{p}$

\begin{tabular}{|c|c|c|c|c|c|c|}
\hline Bearing Number & $d_{p 1}$ & $d_{p 2}$ & $d_{p 3}$ & $d_{p 4}$ & $d_{p 5}$ & $d_{p 6}$ \\
\hline $\mathrm{A}$ & 0.6489 & 0.6485 & 0.5311 & 0.4593 & 0.6542 & 0.6192 \\
\hline $\mathrm{B}$ & 0.6748 & 0.7210 & 0.5377 & 0.6012 & 0.5650 & 0.5797 \\
\hline
\end{tabular}

According to the above table, for bearing $\mathrm{A}$ and $\mathrm{B}$, these are

$$
\begin{aligned}
& d_{p 4}<d_{p 3}<d_{p \sigma} \approx d_{p 2} \approx d_{p 1} \approx d_{p 5} \\
& d_{p 3}<d_{p 5}<d_{p 6}<d_{p 4}<d_{p 1}<d_{p 2}
\end{aligned}
$$

Table 4 Base-based Closeness degree $N$

\begin{tabular}{|c|c|c|c|c|c|c|}
\hline Bearing Number & $N_{1}$ & $N_{2}$ & $N_{3}$ & $N_{4}$ & $N_{5}$ & $N_{6}$ \\
\hline $\mathrm{A}$ & 0.6339 & 0.6015 & 0.7143 & 0.7775 & 0.6264 & 0.6569 \\
\hline $\mathrm{B}$ & 0.5463 & 0.5536 & 0.7246 & 0.6510 & 0.6995 & 0.6993 \\
\hline
\end{tabular}

According to the above table, for bearing A and B, there are 


$$
\begin{aligned}
& N_{4}>N_{3}>N_{6}>N_{1}>N_{5}>N_{2} \\
& N_{3}>N_{5}>N_{6}>N_{4}>N_{2}>N_{1}
\end{aligned}
$$

By using three methods to calculate, different sorting is obtained. The different order of influencing factors of the two bearing vibrations are listed in Table. 5 and Table. 6.

Table 5 The order of vibration influencing factors of bearing A

\begin{tabular}{|c|c|c|}
\hline $\begin{array}{c}\text { Serial } \\
\text { Number }\end{array}$ & Calculation Method & $\begin{array}{c}\text { The order of vibration influencing factors of } \\
\text { bearing }\end{array}$ \\
\hline 1 & Average membership degree & $X_{4} \succ X_{3} \succ X_{6} \succ X_{1} \succ X_{5} \succ X_{2}$ \\
\hline 2 & Euclidean distance & $X_{4} \succ X_{3} \succ X_{6} \succ X_{2} \succ X_{1} \succ X_{5}$ \\
\hline 3 & Base-based Closeness degree & $X_{4} \succ X_{3} \succ X_{6} \succ X_{1} \succ X_{5} \succ X_{2}$ \\
\hline
\end{tabular}

Table 6 The order of vibration influencing factors of bearing B

\begin{tabular}{|c|c|c|}
\hline $\begin{array}{c}\text { Serial } \\
\text { Number }\end{array}$ & Calculation Method & $\begin{array}{c}\text { The order of vibration influencing factors of } \\
\text { bearing }\end{array}$ \\
\hline 1 & Average membership degree & $X_{3} \succ X_{5} \succ X_{6} \succ X_{4} \succ X_{2} \succ X_{1}$ \\
\hline 2 & Euclidean distance & $X_{3} \succ X_{5} \succ X_{6} \succ X_{4} \succ X_{1} \succ X_{2}$ \\
\hline 3 & Base-based Closeness degree & $X_{3} \succ X_{5} \succ X_{6} \succ X_{4} \succ X_{2} \succ X_{1}$ \\
\hline
\end{tabular}

According to qualitative fusion theory, the factors affecting the vibration of the bearing $\mathrm{A}$ are taken the top three - X4, X3 and X6 and the factors affecting the vibration of the bearing B are taken the top three - X3, X5 and X6. According to the intersection operation of set, the main factors affecting the bearing vibration- X3 and X6 are finally determined. Because the order of X1, X2, X4 and X5 is indefinite, it is difficult to distinguish the magnitude of their influence on the bearing vibration.

\section{Conclusions}

The influence degree of the outer ring channel shape error, the inner ring channel surface roughness and structural size on the bearing vibration is small, which indicates that the study of bearing outer ring shape error, the inner ring surface roughness and structural dimensions has reached a relatively high level. Because these influences on the bearing vibration are no longer major factors, so the quality of products for production can maintain the current level. The influence degree of the inner ring channel shape error and the outer ring channel surface roughness on the bearing vibration is large, which indicates that there are still some problems in the design or processing of the bearing. Therefore, the inner ring channel shape error and the outer ring surface roughness become the main factors affecting the bearing vibration[10].

In order to improve bearing performance, the problems of bearing vibration caused by the inner ring channel shape error and the outer ring channel surface roughness should be committed to solve. 


\section{Acknowledgements}

This project is supported by Natural Science Foundation of Henan Province of China (Grant No. 162300410065).

\section{References}

[1] A. Nabhan, M. Nouby, A.M. Sami, and M.O. Mousa: Journal of Low Frequncy Noise Vibration and Active Control, Vol. 35 (2016) No.4, p312-325.

[2] M.A.A. Ismail, N. Sawalhi: Insight, Vol. 59 (2017) No.3, p149-154.

[3] T. Peter W and D. Wang: Sensors, Vol. 17 (2017) No.2.

[4] A. Prudhom, J. Antonino-Daviu, H. Razik and V. Climente-Alarcon: Mechanical Systems and Signal Processing, Vol. 84 (2017), p747-762.

[5] J.F. Guo, J.X. Shi, C.L. Lei and X.C. Wei: Journal of Vibration and Shock, Vol. 34 (2015) No.23, p8-13.

[6] C.M. Pezzani, J.M. Bossio and A.M. Castellino: Mechanical Systems and Signal Processing, Vol. 85 (2017), p354-366.

[7] Q. Wang: Practical Fuzzy Mathematics(Science and Technology Literature Publishing, China 1992), p.13-15. (In Chinese)

[8] Z.Y. Wang, X.T. Xia and J.M. Zhu: Non-Statistical Theory of Measurement Uncertainty( National Defense Industry Press, China 2000). (In Chinese)

[9] J.H. Park, H.E. Kwark and Y.C. Kwun: Journal of Computational Analysis and Applications, Vol. 22 (2017) No.4, p709-725.

[10] X.T. Xia, Z.Y. Wang and H. Chang: Journal of Aeronautical Dynamics, Vol. 20 (2005) No.2, p250-254. 